\title{
Calculation of $\mathrm{TiO}_{2}$ Surface and Subsurface Oxygen Vacancy by the Screened Exchange Functional
}

\author{
Hongfei Li, Yuzheng Guo*, John Robertson \\ Department of Engineering, Cambridge University, \\ Cambridge CB2 1PZ, United Kingdom
}

Abstract

The formation energies of oxygen vacancies at different surface and subsurface sites of anatase (101), anatase (001) and rutile (110) surfaces are calculated by screened-exchange (sX) hybrid functional method. Our results show that the oxygen vacancy is more stable on the surface than subsurface for rutile (110), while it is more stable subsurface than on the surface for anatase surfaces. These results are similar to those found by simple density functional theory, but now the sX hybrid functional gives the correct defect localizations. The defects introduce a gap state near the conduction band edge. For the most stable oxygen vacancy site at each $\mathrm{TiO}_{2}$ surface, the +2 charge state dominates over a wide range of Fermi energies. 


\section{Introduction}

Titanium oxide $\left(\mathrm{TiO}_{2}\right)$ is a technologically important transition metal oxide ${ }^{1}$ widely used in photocatalysis ${ }^{2}$, solar energy conversion ${ }^{3}$, environmental cleanup ${ }^{4}$, and may be in future resistive random-access memories (RRAM) ${ }^{4}$. The oxygen vacancy $\left(V_{O}\right)$ is the most important point defect in $\mathrm{TiO}_{2}$ and it plays a crucial role in these applications. Electron bombardment results confirm that oxygen vacancies rather than Ti interstitials make the dominant contribution to the band gap states of $\mathrm{TiO}_{2}$ surface ${ }^{5}$. Oxygen vacancies also influence the absorption of water and other molecules on $\mathrm{TiO}_{2}$ surfaces in photocatalytic devices ${ }^{6}$. Similarly, oxygen vacancies form a conductive filament across the $\mathrm{TiO}_{2}$ film in RRAM devices ${ }^{4,7,8}$. In addition, the oxygen vacancy usually forms at the surface region of $\mathrm{TiO}_{2}$ in experiments so the surface defects are more important than bulk ones ${ }^{9,10}$. Therefore, it is of great significance to understand the behavior of oxygen vacancies at $\mathrm{TiO}_{2}$ surfaces and associate it with the behavior of $\mathrm{TiO}_{2}$ surfaces and devices.

While rutile is the thermodynamically most stable bulk phase of $\mathrm{TiO}_{2}$, anatase is more stable for particles below $\sim 14 \mathrm{~nm}$ in size due to its lower surface energies ${ }^{11}$. Thus, anatase is more relevant to catalytic applications. There has been a continuing interest in the properties of anatase and rutile surfaces ${ }^{1}$. Rutile (110), its most stable surface, has a high surface energy $\left(0.82 \mathrm{~J} / \mathrm{m}^{2}\right)$, whereas anatase has a much lower surface energy of $0.44 \mathrm{~J} / \mathrm{m}^{2}$ for its most stable (101) facet. The more reactive (001) facet of anatase is calculated to have a surface energy of $\sim 0.90 \mathrm{~J} / \mathrm{m}^{2}$. However, this 
surface can undergo a surface reconstruction to give the ad-molecule (ADM) reconstruction, as confirmed by $\mathrm{STM}^{12}$, with a calculated surface energy of $0.51 \mathrm{~J} / \mathrm{m}^{2}$. This minority $\mathrm{TiO}_{2}$ facet has aroused much attention since it was fabricated by stabilizing with a hydrofluoric acid morphology controlling agent ${ }^{13}$.

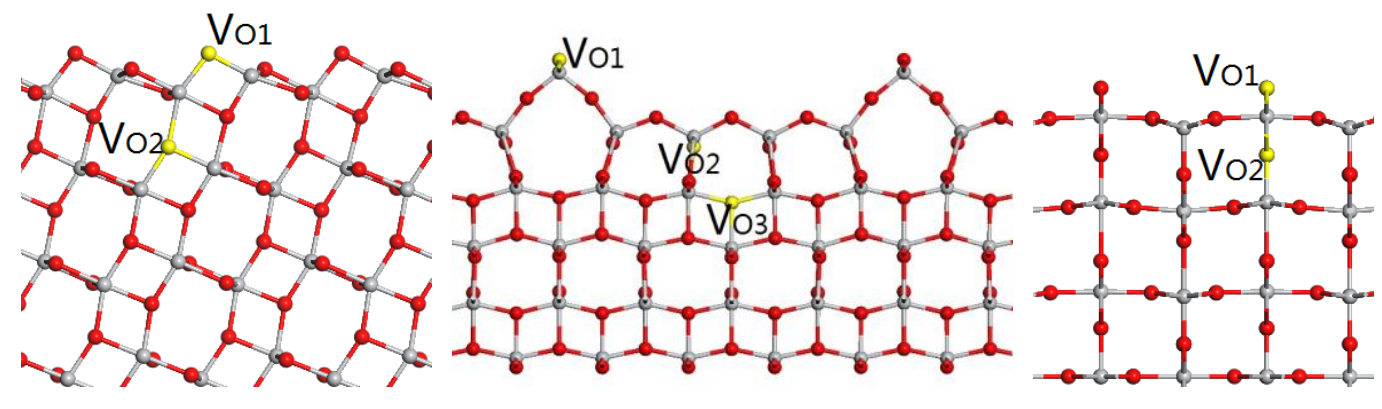

(a)

(b)

(c)

Fig 1. Slab models of (a) the anatase (101) surface, (b) anatase (001) surface and (c) rutile (110) surface. Oxygen is red, Titanium is grey. The various oxygen vacancy sites are yellow.

As $\mathrm{O}$ vacancies mediate the reactivity of $\mathrm{TiO}_{2}$ surfaces, it is expected that the surface with the higher vacancy concentration might be the most reactive. However, resonant photoemisssion experiments ${ }^{14}$ suggest that anatase has a relatively low vacancy concentration. Cheng and Selloni ${ }^{15}$ explained this by showing that the $\mathrm{O}$ vacancy is more stable subsurface in anatase, whereas it is more stable on the surface in rutile. Nevertheless, these results were obtained by the simple generalized gradient approximation (GGA) version of density functional theory with rather small 
supercells. It is well known that that local functionals such as GGA under-estimates the band gap in semiconductors and insulators and does not handle the Coulomb interaction in strongly correlated systems well ${ }^{16,17}$. In $\mathrm{TiO}_{2}$ this has the effect that local functionals under-estimate the $d$ electron localization and the amount of lattice distortions at vacancies. They under-estimate the depth of their defect states in the band gap ${ }^{18,19}$. Thus, it is unclear if predictions of vacancy behavior between the bulk and surfaces in $\mathrm{TiO}_{2}$ based only on GGA are fully reliable. It is therefore worthwhile to check these results using advanced methods that correct these errors. The simplest method would be to use DFT $+\mathrm{U}$ which includes an on-site repulsion potential $\mathrm{U}^{20}$. However, it requires rather unphysical values of $\mathrm{U}$ in the case of $\mathrm{TiO}_{2}$ to give a correct band gap. Thus, it is preferable to use hybrid functional such as B3LYP, the Heyd, Scuseria, Erzernhof (HSE) functional ${ }^{21}$ or screened exchange (sX) functional ${ }^{22}$ which give good representations of the electronic structure. They are also computationally efficient for large supercells of 100-200 atoms needed for defect calculation. The sX hybrid functional method was found to give the accurate band gaps of many semiconductors and insulators and the correct localization of charge near vacancies $^{23-27}$.

We have previously presented the results for oxygen vacancies in bulk rutile by $\mathrm{sX}^{28}$. Similar results using B3LYP, HSE and GW methods are also available ${ }^{18,19,29-33}$. Here we present the results for the surface oxygen vacancies in $\mathrm{TiO}_{2}$ by $\mathrm{sX}$. To this end, we calculate the formation energy of oxygen vacancies at three surfaces, anatase 
(101), reconstructed anatase (001), and rutile (110) by the sX hybrid functional to find out the relative stability of the surface and subsurface positions. In this paper we also include the results of $\mathrm{GGA}^{34}$ for comparison. It should be noted that B3LYP gives band gaps larger than experiments for rutile ${ }^{19}$, which might have lead to stronger defect state localization. Similarly, HSE gives the correct distortions around the bulk $\mathrm{O}$ vacancy in rutile. But the defect level lies at the conduction band edge rather than deeper into the gap as seen experimentally ${ }^{29}$. In contrast, sX gives the correct band gap for rutile and gave the correct vacancy distortions and a deep state for the vacancy $^{28}$.

\section{Methods}

The GGA and screened exchange hybrid functional calculations are carried out using the CASTEP plane wave pseudopotential $\operatorname{code}^{35}$. For $\mathrm{k}$ sampling we use the $\Gamma$ point scheme due to the large supercell. The geometry optimization is carried out until the residual force is smaller than $0.01 \mathrm{eV} / \AA$.

The three $\mathrm{TiO}_{2}$ surfaces we constructed are shown in Fig 1. We used slabs of four $\mathrm{TiO}_{2}$ layers containing 144 atoms for the anatase (101) surface $(2 \times 3$ surface supercell), and slabs of four $\mathrm{TiO}_{2}$ layers containing 192 atoms for the rutile (110) surface $(4 \times 2$ surface supercell). For the anatase $(001)$ surface, we took the ADM model ${ }^{12}$ as introduced above, with five $\mathrm{TiO}_{2}$ layers containing 189 atoms $(4 \times 3$ surface supercell). All of the supercell lattice constants are larger than $11 \AA$ in all of the three 
directions. Our previous study showed that the $\mathrm{O}$ vacancy in $\mathrm{TiO}_{2}$ has strong polaronic effects ${ }^{36}$ and thus requires a larger supercell to suppress the defect image interaction $^{28}$. Further calculations of the $\mathrm{O}$ vacancy with a larger $3 \times 3 \times 4$ supercell of rutile than in Lee et $\mathrm{al}^{28}$ find that the $\mathrm{V}_{\mathrm{O}}^{+1}$ state distorts such that the unpaired electron is localized on one of the 3 adjacent sites, and therefore in agreement with recent electron spin resonance data ${ }^{37}$. All surfaces were modeled using the periodic boundary conditions with a vacuum of $\sim 11 \AA$.

The defect properties are represented by their formation energies as a function of Fermi energy and chemical potential. The charge correction follows Lany and Zunger's scheme ${ }^{38}$. The formation energy $\mathrm{H}_{\mathrm{q}}$ of defect with charge $\mathrm{q}$ can be calculated by following expression ${ }^{38}$

$$
\mathrm{H}_{\mathrm{q}}\left(\mathrm{E}_{\mathrm{F}}, \mu\right)=\left[\mathrm{E}_{\mathrm{q}}-\mathrm{E}_{\mathrm{H}}\right]+\mathrm{q}\left(\mathrm{E}_{\mathrm{V}}+\Delta \mathrm{E}_{\mathrm{F}}\right)+\sum_{\alpha} \mathrm{n}_{\sigma}\left(\mu_{\alpha}\right)
$$

where $E_{q}$ and $E_{H}$ are the total energy of a defect cell and a perfect cell with charge $q$ respectively, $\Delta \mathrm{E}_{\mathrm{F}}$ is the Fermi level energy with respect to valence band maximum, $\mathrm{n}_{\alpha}$ is the number of atoms of element $\alpha$, and $\mu_{\alpha}$ is reference chemical potential of element $\alpha$. The chemical potentials satisfy the following equation (experimental value),

$$
\mu_{\mathrm{Ti}}+2 \mu_{\mathrm{O}}=\mathrm{H}_{\mathrm{f}}\left(\mathrm{TiO}_{2}\right)=-9.73 \mathrm{eV}
$$

The O-rich limit is at $\mu_{\mathrm{O}}=0 \mathrm{eV}$, and $\mu_{\mathrm{Ti}}=-9.73 \mathrm{eV}$. The O-poor limit (or Ti-rich limit) is not the usual $\mathrm{Ti}: \mathrm{TiO}_{2}$ equilibrium but the $\mathrm{Ti}_{2} \mathrm{O}_{3}: \mathrm{TiO}_{2}$ equilibrium ${ }^{28,29}$,

$$
2 \mu_{\mathrm{Ti}}+3 \mu_{\mathrm{O}}=\mathrm{H}_{\mathrm{f}}\left(\mathrm{Ti}_{2} \mathrm{O}_{3}\right)=-15.39 \mathrm{eV}
$$


Therefore, $\mu_{\mathrm{O}}=-\mathrm{H}_{\mathrm{f}}\left(\mathrm{Ti}_{2} \mathrm{O}_{3}\right)+2 \mathrm{H}_{\mathrm{f}}\left(\mathrm{TiO}_{2}\right)$ and $\mu_{\mathrm{Ti}}=2 \mathrm{H}_{\mathrm{f}}\left(\mathrm{Ti}_{2} \mathrm{O}_{3}\right)-3 \mathrm{H}_{\mathrm{f}}\left(\mathrm{TiO}_{2}\right)$, which gives $\mu_{\mathrm{O}}=-4.07 \mathrm{eV}$ and $\mu_{\mathrm{Ti}}=-1.59 \mathrm{eV}^{28}$.

\section{Results}

The local density of states (DOS) of the three kinds of $\mathrm{TiO}_{2}$ surfaces are shown in the Fig. 2 and compared to the bulk $\mathrm{TiO}_{2}$ DOS. The calculated bulk $\mathrm{TiO}_{2}$ band gaps are $3.02 \mathrm{eV}$ for rutile and $3.17 \mathrm{eV}$ for anatase, close to the experimental value. The dangling bonds on the surface introduced the gap states expanding into the band gap of bulk $\mathrm{TiO}_{2}$. The surface band gaps are thus smaller than the bulk value, $3.11 \mathrm{eV}$ for anatase (101), $2.76 \mathrm{eV}$ for anatase $(001)$ and $2.90 \mathrm{eV}$ for rutile (110). Our simulation results are consistent with the experimental surface band gap values in previous studies $^{39,40}$

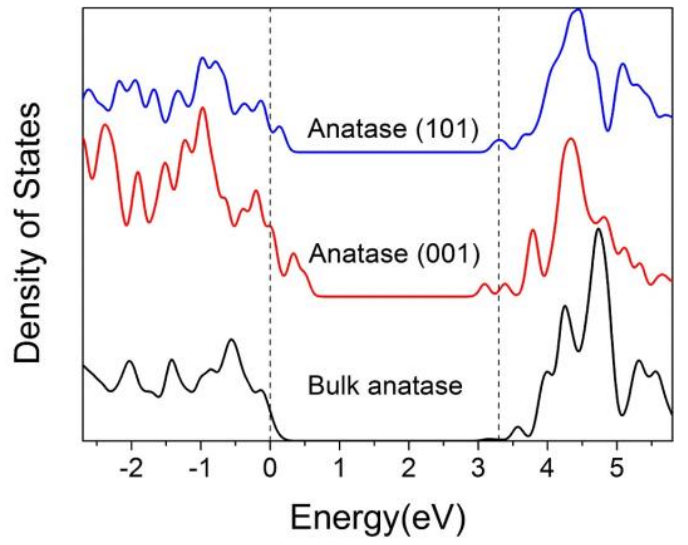

(a)



(b)

Fig 2. DOS of (a) anatase (101) surface, anatase (001) surface and bulk anatase; (b) rutile (110) surface and bulk rutile, calculated by sX-LDA method. The dashed line marks the conduction band minimum and valance band maximum of bulk $\mathrm{TiO}_{2}$. The 
O $2 \mathrm{~s}$ orbital at $-20 \mathrm{eV}$ below Fermi level is used as a reference level to align the bulk and interface DOS.

We have calculated the formation energy of various surface or subsurface neutral oxygen vacancies on all three surfaces in the O-rich condition, as labeled in Fig. 1, in both GGA and screened exchange. The most stable ones are listed in Table 1. The formation energy of the oxygen vacancy in bulk $\mathrm{TiO}_{2}$ is also listed there for comparison.

First we discuss the most stable oxygen vacancies at the anatase (101) surface. The surface defect site $\mathrm{V}_{\mathrm{O} 1}$ corresponds to the removal of an $\mathrm{O}_{2 \mathrm{C}}$, while $\mathrm{V}_{\mathrm{O} 2}$, the subsurface defect site, corresponds to the removal of an $\mathrm{O}_{3 \mathrm{C}}$. Both GGA and sX find that $\mathrm{V}_{\mathrm{O} 2}$ is more favorable, with an energy difference of $0.3 \mathrm{eV}$ for $\mathrm{sX}$ and $0.4 \mathrm{eV}$ for GGA. This result is consistent with the quite low surface energy of anatase (101). Thus it takes more energy to create a surface vacancy in anatase. In addition, the subsurface oxygen vacancy formation energy is only $0.17 \mathrm{eV}$ less than that in bulk anatase from sX. Hence the oxygen vacancy is more likely to form at anatase (101) subsurface rather than at the surface or bulk site. 
Table 1. Formation energies of neutral oxygen vacancy defect at different sites of anatase (101), anatase (001), and rutile (110) surfaces. Positive value stands for the extra energy needed to form defect.

\begin{tabular}{|c|l|r|r|}
\hline Formation Energy & Vacancy Site & \multicolumn{1}{|c|}{$\mathrm{GGA}(\mathrm{eV})$} & \multicolumn{1}{c|}{$\mathrm{sX}-\mathrm{LDA}(\mathrm{eV})$} \\
\hline \multirow{4}{*}{ anatase (101) } & $\mathrm{V}_{\mathrm{O} 1}$ (Surface) & 4.37 & 5.08 \\
\cline { 2 - 4 } & $\mathrm{V}_{\mathrm{O} 2}$ (Subsurface) & $\mathbf{3 . 9 9}$ & $\mathbf{4 . 7 8}$ \\
\cline { 2 - 4 } & $\mathrm{V}_{\mathrm{O}}$ in bulk & 4.27 & 4.95 \\
\hline \multirow{5}{*}{ anatase (001) } & $\mathrm{V}_{\mathrm{O} 1}$ (Surface) & 4.74 & 5.07 \\
\cline { 2 - 4 } & $\mathrm{V}_{\mathrm{O} 2}$ (Surface) & 4.38 & 5.12 \\
\cline { 2 - 4 } & $\mathrm{V}_{\mathrm{O}}$ (Subsurface) & 4.21 & 4.96 \\
\cline { 2 - 4 } & $\mathrm{V}_{\mathrm{O}}$ in bulk & 4.27 & 4.95 \\
\hline \multirow{4}{*}{ rutile (110) } & $\mathrm{V}_{\mathrm{O} 1}$ (Surface) & 3.71 & 4.39 \\
\cline { 2 - 4 } & $\mathrm{V}_{\mathrm{O} 2}$ (Subsurface) & 4.10 & 4.98 \\
\cline { 2 - 4 } & $\mathrm{V}_{\mathrm{O}}$ in bulk & 4.79 & 5.70 \\
\hline
\end{tabular}

The bold denotes the most stable defect site for each surface. The values are compared with oxygen vacancy in bulk $\mathrm{TiO}_{2}$. Site labels are given in Fig. 1 .

We then analyze the three most stable oxygen vacancies on anatase (001). $\mathrm{V}_{\mathrm{O} 1}$ is at the ridge part of the surface, which is originally bonded to two Ti atoms. $\mathrm{V}_{\mathrm{O} 2}$, corresponding to the removal of an $\mathrm{O}_{3 \mathrm{C}}$, is at the terrace part of the surface, and $\mathrm{V}_{\mathrm{O} 3}$ is at the subsurface, which is originally bonded to three Ti atoms. Both GGA and sX show that the subsurface oxygen vacancy $\mathrm{V}_{\mathrm{O} 3}$ is most stable, similar to anatase (101). In addition, the formation energy of the oxygen vacancy in bulk anatase is $4.95 \mathrm{eV}$, which is nearly the same as that of $\mathrm{V}_{\mathrm{O} 3}$ in anatase (001) surface, while the formation energy of $\mathrm{V}_{\mathrm{O} 2}$ in anatase (101) is $0.2 \mathrm{eV}$ smaller. Thus, the subsurface oxygen vacancy in anatase (001) experiences an environment similar to the bulk $\mathrm{TiO}_{2}$ and the oxygen vacancy is likely to form at subsurface and bulk site rather than surface. Furthermore, 
we can also conclude that $\mathrm{O}$ vacancies slightly prefer to form at the subsurface rather than the top surface of anatase (101) and (001).

For rutile (110), Table 1 lists the two most stable oxygen vacancies that we find in our calculations. $\mathrm{V}_{\mathrm{O} 1}$ is at the surface, which is originally bonded to two Ti atoms while $\mathrm{V}_{\mathrm{O} 2}$ is a subsurface defect corresponding to the removal of an $\mathrm{O}_{3 \mathrm{C}}$ atom. Both GGA and sX show that $\mathrm{V}_{\mathrm{O} 1}$ is the most stable defect with a formation energy of $1.3 \mathrm{eV}$ lower than $\mathrm{V}_{\mathrm{O}}$ in bulk rutile. Thus, oxygen vacancies are more likely to form at the rutile surface. This result can be interpreted as that it is only necessary to break two Ti-O bonds to create an oxygen vacancy at the rutile (110) surface rather than to break three Ti-O bonds at subsurface or bulk. This is quite different from anatase (101) or (001) where the subsurface defect is quite similar to the bulk case.

Removal of an oxygen atom in the $\mathrm{TiO}_{2}$ surface structure creates two unpaired electrons (for the neutral defect) and dangling bonds on adjacent Ti atoms. Fig. 3(a) shows that on the anatase (101) surface removal of the oxygen atom at $\mathrm{V}_{\mathrm{O} 2}$ shifts the adjacent Ti atoms away from the defect and nearby oxygen atoms towards the defect. The Ti-Ti distance increases from $3.20 \AA$ to $3.70 \AA$ and from $4.00 \AA$ to $4.75 \AA$ and a Ti-O bond near the defect breaks due to the distortion. Fig. 3(b) shows that on anatase (001) surface the removal of an oxygen atom at the $\mathrm{V}_{\mathrm{O} 3}$ site also causes adjacent $\mathrm{Ti}$ atoms to move apart and $\mathrm{O}$ atoms to move closer to defect. The Ti-Ti distance between adjacent $\mathrm{Ti}$ atoms increases from $3.77 \AA$ to $4.12 \AA$ and from $3.08 \AA$ to $3.43 \AA$ 
and the $\mathrm{O}-\mathrm{O}$ distance between nearby $\mathrm{O}$ atoms decrease from $3.74 \AA$ to $3.34 \AA$. Fig. 3(c) shows that for the rutile (110) surface, the lowest cost $O$ vacancy is at the surface. Removing an oxygen atom only forms two Ti dangling bonds. This not only causes the adjacent $\mathrm{Ti}$ atoms to move away from defect, increasing the separation from $2.96 \AA$ to $3.37 \AA$, but also triggers these two Ti atoms to move $0.40 \AA$ downwards into bulk. However, the next-nearest $\mathrm{Ti}$ atoms stay nearly unchanged, thus the oxygen vacancy only leads to a local deformation in $\mathrm{TiO}_{2}$. 
(a)


(b)
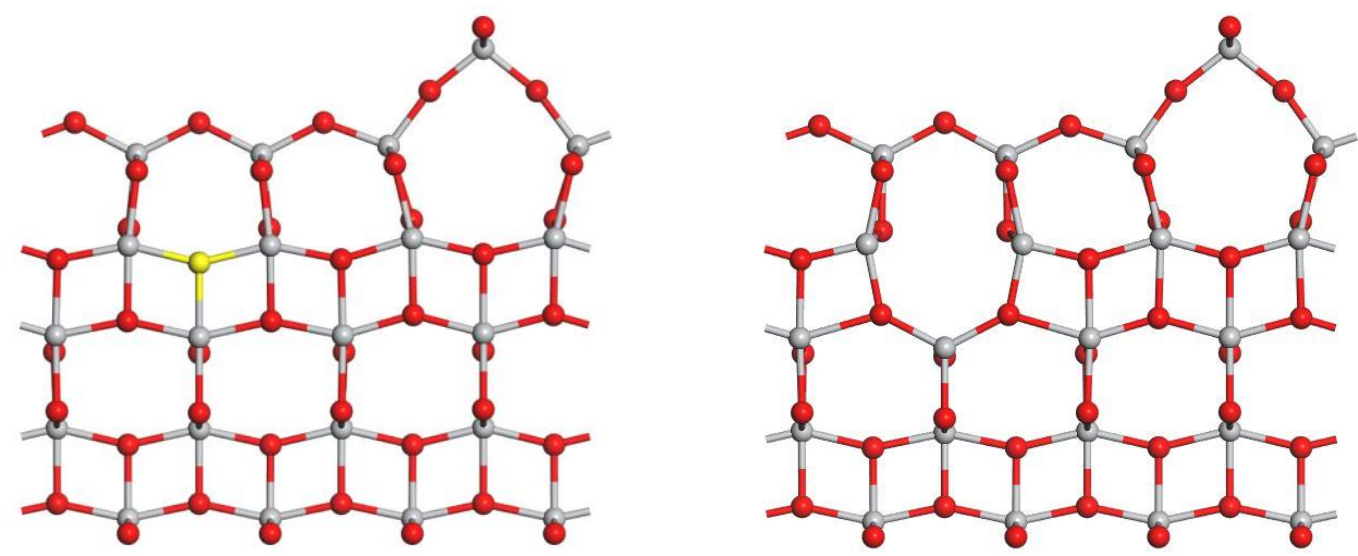

(c)
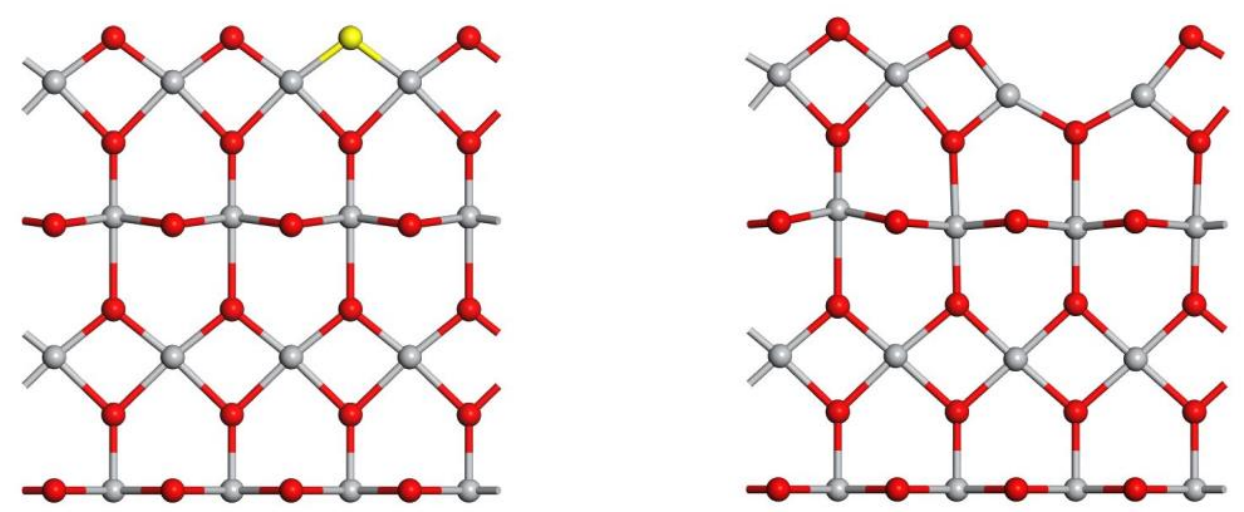

Fig 3. Side view of atomic spacing of (a) anatase (101) surface, (b) anatase (001) surface and (c) rutile (110) surface. The figures on the left are perfect slabs without 
defect, and the figures on the right are the surface structure with an oxygen vacancy after geometry relaxation.

Fig 4 shows the calculated formation energies of most stable oxygen vacancies at these three surfaces for both O-poor and O-rich conditions by sX, as a function of the Fermi energy. Defect charge states from -2 to 2 have been calculated. Possible spin polarization is also included. The $\mathrm{E}_{\mathrm{C}}$ and $\mathrm{E}_{\mathrm{V}}$ here refer to the conduction band and valence band edge of the $\mathrm{TiO}_{2}$ surface system. The surface band gap is smaller than bulk value. The charge transition levels correspond to the Fermi energy at which the charge q and q' defect states have the same formation energy. The defect charge state would change at these energies.

On the anatase (101) surface, the most stable oxygen vacancy $\mathrm{V}_{\mathrm{O} 2}$ is mostly dominated by the +2 charge state, and the calculated $\mathrm{V}_{\mathrm{O}}{ }^{+} / \mathrm{V}_{\mathrm{O}}{ }^{2+}$ charge transition is just $0.1 \mathrm{eV}$ below the conduction band minimum (CBM). At the O-rich limit, the formation energy of $\mathrm{V}_{\mathrm{O}}{ }^{0}$ is $4.60 \mathrm{eV}$. On the anatase (001) surface, the formation energy of $\mathrm{V}_{\mathrm{O} 3}{ }^{0}$ is $4.96 \mathrm{eV}$ when $\mathrm{E}_{\mathrm{F}}$ is near the conduction band edge. The $\mathrm{V}_{\mathrm{O}}{ }^{0} / \mathrm{V}_{\mathrm{O}}{ }^{2+}$ charge transition is at $0.2 \mathrm{eV}$ below the conduction band. The +1 charge state is unstable. So it is a negative $\mathrm{U}$ system ${ }^{41}$. For the rutile (110) surface, the most stable surface defect $\mathrm{V}_{\mathrm{O} 1}{ }^{0}$ has the formation energy of $4.39 \mathrm{eV}$ when $\mathrm{E}_{\mathrm{F}}$ is near the conduction band edge, while the $2+$ charge state is most stable for $\mathrm{E}_{\mathrm{F}}$ lying lower in the band gap. 
(a)
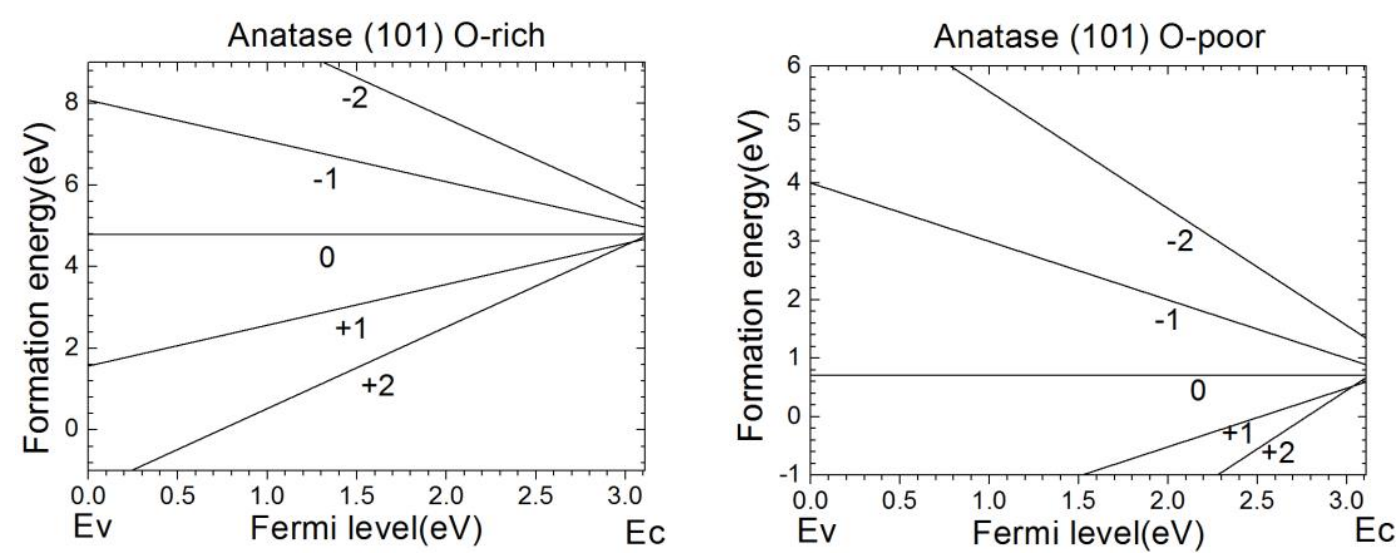

(b)
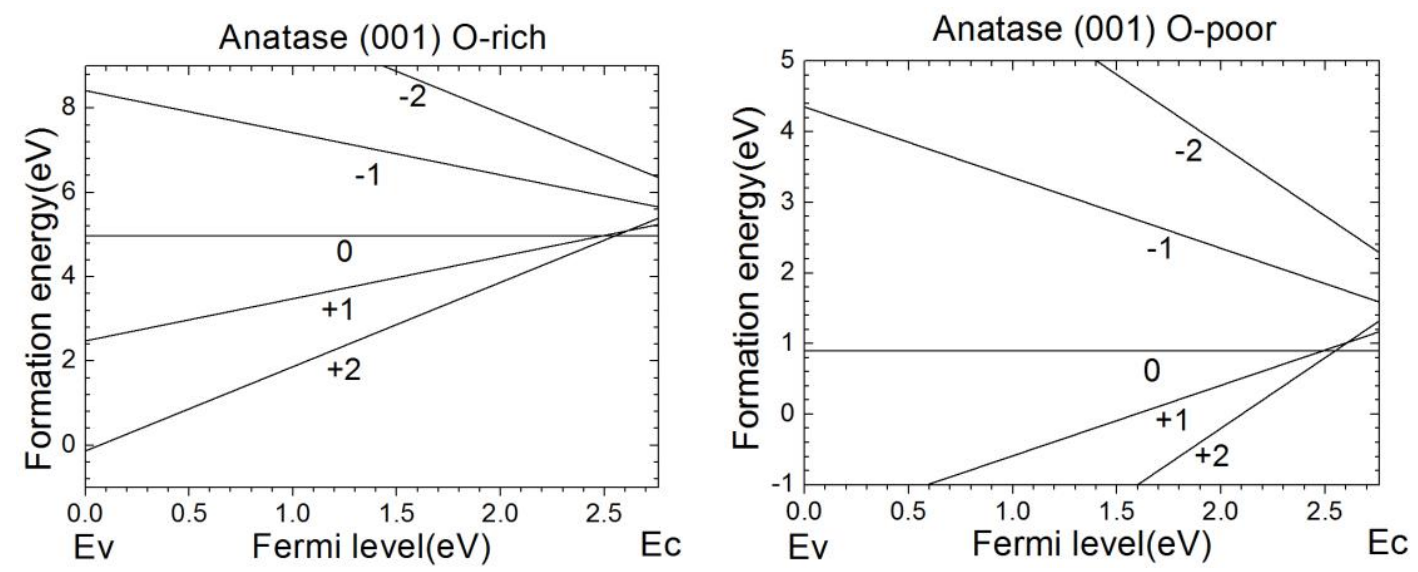

(c)
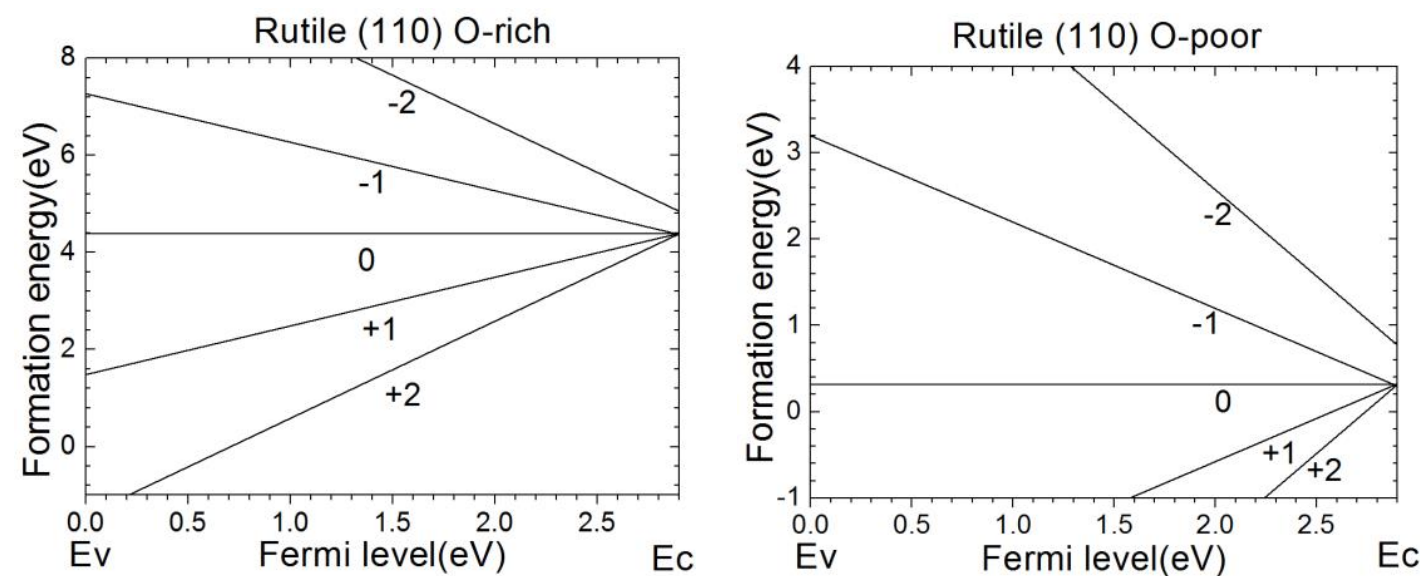

Fig 4. The oxygen vacancy formation energy against Fermi level at O-rich and O-poor conditions, calculated by sX-LDA for (a) anatase (101), (b) anatase (001), and 
(c) rutile (110) surface. $E_{C}$ and $E_{V}$ refer to the conduction band and valance band edge of $\mathrm{TiO}_{2}$ surface. Negative charge states are also shown for completeness.

Fig 5 shows the partial density of states (PDOS) of the most stable defect sites for $\mathrm{V}_{\mathrm{O}}{ }^{2+}$ charge state on the three surfaces. Here we compared the total DOS, the PDOS on Ti atoms near defect and in the bulk, as well as the PDOS on O atoms in bulk. In sX, the oxygen vacancy gives rise to a defect state just above CBM on anatase (101) and anatase (001) surface, while a defect state appears below CBM on rutile (110) surface. 


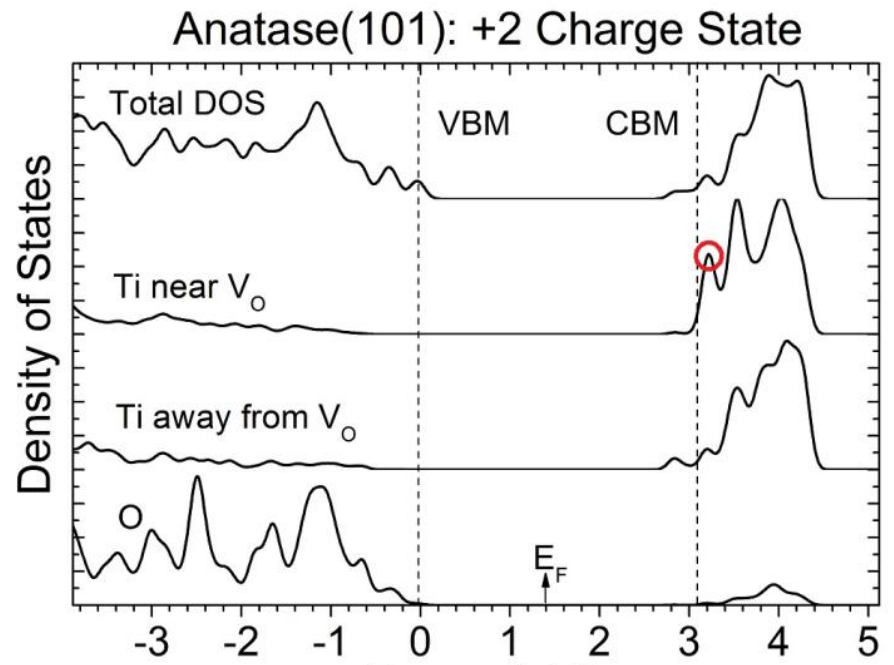

(a)

Energy $(\mathrm{eV})$

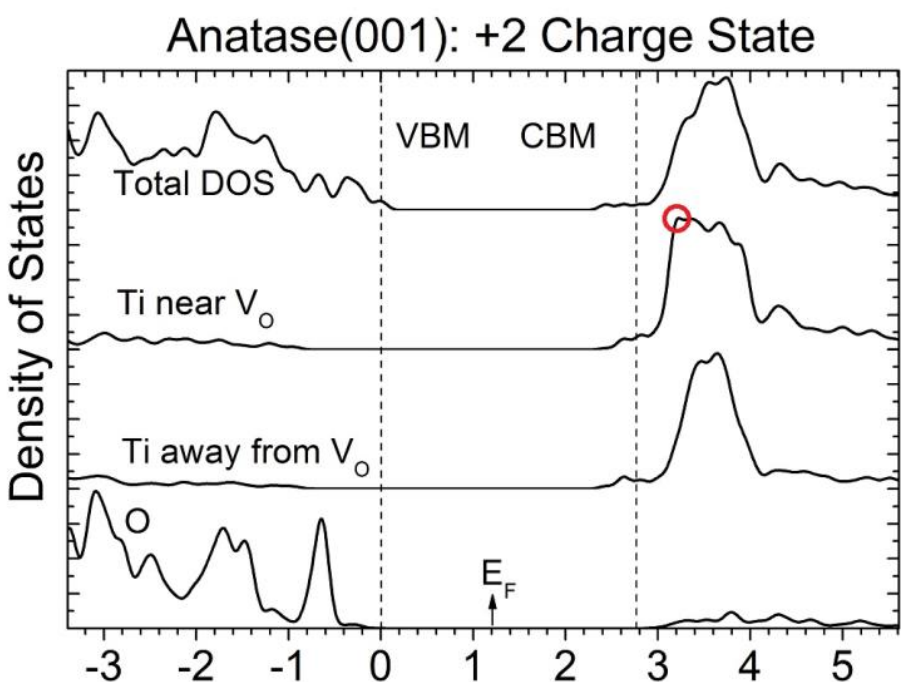

(b)

Energy(eV)

Rutile(110): +2 Charge State

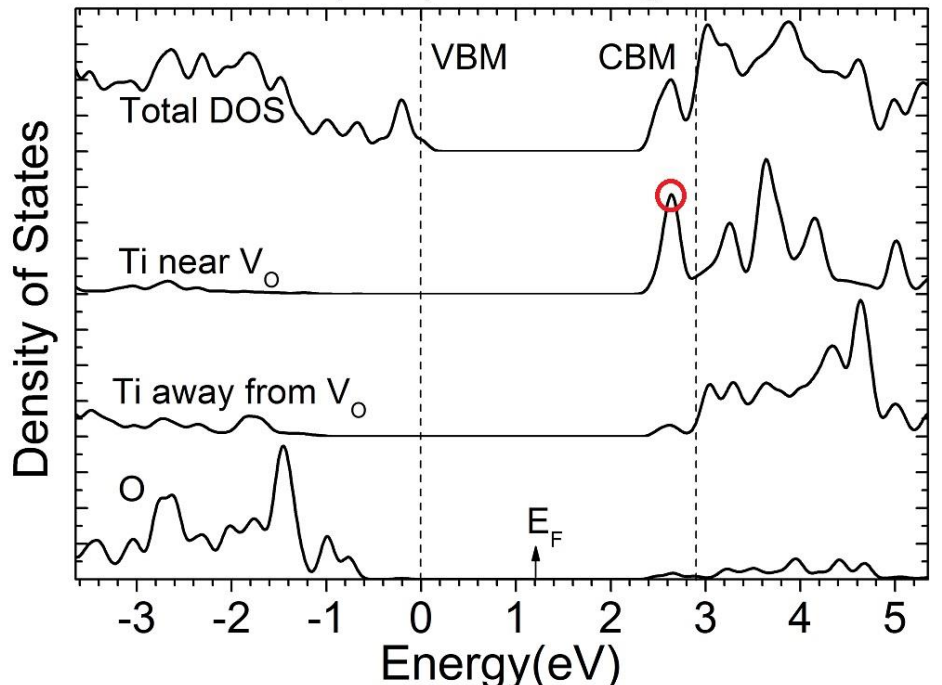

(c) Energy $(\mathrm{eV})$ 
Fig 5. The partial density of states (PDOS) of the three $\mathrm{TiO}_{2}$ surfaces with an oxygen vacancy at +2 charge states, (a) anatase (101) surface, (b) anatase (001) surface, and (c) rutile (110) surface, by sX-LDA method. The PDOS on Ti atom near the defect, on Ti atom away from defect and on $\mathrm{O}$ atom are shown here and compared with total DOS. The VBM and CBM are calculated from the perfect surface slab model and aligned to the PDOS of defect system. The defect peak is indicated with red circle in each figure.

The charge density for $\mathrm{V}_{\mathrm{O} 2}{ }^{0}, \mathrm{~V}_{\mathrm{O} 2}{ }^{+}$, and $\mathrm{V}_{\mathrm{O} 2}{ }^{2+}$ defects on anatase (101) are shown in Fig 6. (a), (b), (c) separately from the same direction. Different charge states of the defect trigger different lattice distortions on adjacent sites. Interestingly, the charge density is localized on two Ti atoms rather than the defect site. One of the Ti atoms is the nearest neighbor and the other is the next-nearest neighbor. Both of them are at the surface. This is different from that in bulk $\mathrm{TiO}_{2}$ as described in $\mathrm{ref}^{28}$, due to the influence of the surface. However, this localization scheme is consistent with the B3LYP and PBE+U results of Di Valentin et $a l^{19}$. Recent studies on the anatase (101) surface show that the surface induced 5-fold Ti can introduce a strong polaronic effect and localize the excess electrons near the surface ${ }^{42,43}$. We have also calculated the charge density of defect level on anatase (001) and rutile (110) surface. Both of them are delocalized and not shown here. 




(a)

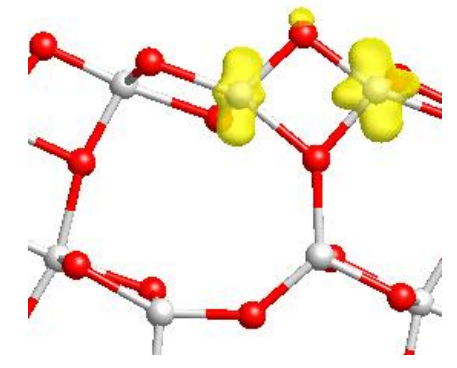

(b)



(c)

Fig 6. The charge density contour for oxygen vacancy near CBM of anatase (101) surface: (a) $\mathrm{V}_{\mathrm{O} 2}{ }^{0}$, (b) $\mathrm{V}_{\mathrm{O} 2}{ }^{+}$, (c) $\mathrm{V}_{\mathrm{O} 2}{ }^{2+}$

\section{Discussion}

Various experimental studies have confirmed the existence of oxygen vacancies on the surface of rutile (110), which are in good agreement with our simulation results. Fukui et $a l^{9}$ directly observed the oxygen vacancies on rutile (110) surface by AFM . Wendt $e{ } a{ }^{10}$ also confirmed the existence of oxygen vacancies on this surface by STM. Both AFM and STM figures of surface oxygen vacancies support our simulation results that oxygen vacancies are more likely to form on the surface of rutile (110), since the formation energy of oxygen vacancy at rutile (110) surface is smaller than that at subsurface and much smaller than that in bulk rutile.

We compare our sX simulation results of the neutral $V_{O}$ formation energy with the GGA results by Cheng et $a l^{15}$. Both GGA and sX show that subsurface Vo is the most 
stable for the two anatase surfaces, while the surface $V_{O}$ is the most stable for rutile (110) surfaces.

We also take larger supercells for all three surface models than in Cheng et $a l^{15}$. In our simulation, a distance of at least $10 \AA$ is ensured between the defect and its image in all directions and the slab model is thick enough along $\mathrm{z}$ direction to eliminate the influence of the bottom surface. Our cell size tests indicate that a smaller supercell can lead to an incorrect order of formation energies. For the anatase (001) surface, the four slabs supercell we have tested is not thick enough that the subsurface $\mathrm{V}_{\mathrm{O} 3}$ could be influenced by both the top surface and the bottom surface in the model, which results in an unreasonable charge distribution and formation energy.

We also compare our results of Vo on the surface and in the bulk with other advanced methods beyond GGA such as GW. It is widely agreed that the neutral Vo formation energy in bulk rutile at $\mathrm{O}$-poor limit is around $1.8 \mathrm{eV}$, which is also confirmed by our simulation. However, the +2 charge state of Vo formation energy differs largely in different work. The HSE result given by Janotti et $a l^{29}$ suggests this value to be $-5.1 \mathrm{eV}$ while the $\mathrm{GW}$ result of Malashevich et $a l^{30}$ gives the formation energy of $-3.7 \mathrm{eV}$. Our $\mathrm{sX}$ results give $-4.30 \mathrm{eV}$, which is in the reasonable region. By comparing the oxygen vacancy formation energy on the surface and in the bulk, we find that anatase has a similar formation of Vo on surface as it is in bulk, while rutile Vo on the surface is thermodynamically much preferred than in bulk Vo. Thus, $\mathrm{V}_{\mathrm{O}}$ is 
likely to form in the surface area, while for the anatase surface the oxygen vacancy tends to form at the subsurface and bulk region.

Our simulation also shows that formation energy of oxygen vacancy at anatase (101) subsurface is smaller than that at anatase (001). The $\mathrm{O}$ vacancy formation probability is roughly the same for all sites (including the surface ones) with only slight preference for sub-surface or bulk regions of anatase(101) or (001) surfaces. So the surface and subsurface region does not possess any advantage over bulk for the oxygen vacancy's formation. These two mechanisms jointly explain the reason for a much lower oxygen vacancy concentration on the anatase (001) surface than the anatase (101) surface as indicated by resonant photoemission data ${ }^{14}$.

It is also interesting to use our results for interpreting oxygen vacancy behavior in RRAM. In this case, a scavenging metal layer is usually included, so that the system is in the $\mathrm{O}$ poor limit. In addition, the electrode metals are chosen to set the resting Fermi energy. $\mathrm{E}_{\mathrm{F}}$ will tend to lie in the range of $4.3 \mathrm{eV}$ to $5.3 \mathrm{eV}$ below vacuum, or $0.4-1.4 \mathrm{eV}$ below the bulk conduction band edge. In the O-poor limit, the neutral $\mathrm{O}$ vacancy formation energy becomes quite low, below $1 \mathrm{eV}$. When $\mathrm{E}_{\mathrm{F}}$ is lowered to that the vacancy enters its $2+$ charge state, its formation energy becomes negative. The Fermi level cannot move further towards the lower part of band gap ${ }^{44}$. This is indeed what happens in the devices, and the vacancies then coalesce to form the conductive filament. 


\section{Conclusions}

We calculate the electronic structure of oxygen vacancies with different charge states on three $\mathrm{TiO}_{2}$ by screened exchange functional. On all of the three surfaces, the +2 charge state is the most stable for oxygen vacancy in most part of band gap. The formation energy of Vo is negative near VBM at O-rich limit so that $\mathrm{O}$ vacancy could form spontaneously at low Fermi level or O poor condition. This is favorable to the formation of the conducting channel in RRAM. The energy differences among various sites are small in anatase with slightly preference for sub-surface while the rutile surface site has much lower formation energy. For all three $\mathrm{TiO}_{2}$ surface models, oxygen vacancies introduce a gap state near the conduction band edge. The defect orbital is localized on a neighboring Ti atom and a next-nearest Ti atom, which is different from bulk $\mathrm{TiO}_{2}$ defect. 
References

1 Diebold, U. The Surface Science of Titanium Dioxide. Surf. Sci. Rep. 2003, $48,53-229$.

2 Asahi, R.; Morikawa, T.; Ohwaki, T.; Aoki, K.; Taga, Y. Visible-light Photocatalysis in Nitrogen-doped Titanium Oxides. Science 2001, 293, 269-271.

3 M. Gratzel. Photoelectrochemical Cells. Nature 2001, 414, 338-344.

4 Kwon, D. H.; Kim, K. M.; Jang, J. H.; Jeon, J. M.; Lee, M. H.; Kim, G. H.; Li, X. S.; Park, G. S.; Lee, B.; Han, S.; et al. Atomic Structure of Conducting Nanofilaments in $\mathrm{TiO}_{2}$ Resistive Switching Memory. Nature Nanotech 2010, 5, $148-153$.

5 Yim, C. M.; Pang, C. L.; Thornton, G. Oxygen Vacancy Origin of the Surface Band-Gap State of $\mathrm{TiO}_{2}$ (110). Phys. Rev. Lett. 2010, 104, 036806 1-4.

6 Schaub, R.; Thostrup, P.; Lopez, N.; Laegsgaard, E.; Stensgaard, I.; Norskov, J. K.; Besenbacher, F. Oxygen Vacancies as Active Sites for Water Dissociation on Rutile $\mathrm{TiO}_{2}$ (110). Phys. Rev. Lett. 2001, 87, 266104 1-4.

7 Park, S. G.; Magyari-Kope, B.; Nishi, Y. Impact of Oxygen Vacancy Ordering on the Formation of a Conductive Filament in $\mathrm{TiO}_{2}$ for Resistive Switching Memory. IEEE ED Lett. 2011, 32, 197-199.

8 Guo, Y.; Robertson, J. Materials Selection for Oxide-Based Resisitive Random Access Memories. Appl. Phys. Lett. 2014, 105, 223516 1-5. 
9 Fukui, K.; Onishi, H.; Iwasawa, Y. Atom-resolved Image of the $\mathrm{TiO}_{2}(110)$

Surface by Noncontact Atomic Force Microscopy. Phys. Rev. Lett. 1997, 79, 4202-4205.

10 Wendt, S.; Schaub, R.; Matthiesen, J.; Vestergaard, E. K.; Wahlstrom, E.;

Rasmussen, M. D.; Thostrup, P.; Molina, L. M.; Laegsgaard, E.; Stensgaard, I.; et al. Oxygen Vacancies on $\mathrm{TiO}_{2}(110)$ and their Interaction with $\mathrm{H}_{2} \mathrm{O}$ and $\mathrm{O}_{2}$ : A Combined High-resolution STM and DFT Study. Surf. Sci. 2005, 598, 226-245.

11 Ranade, M. R.; Navrotsky, A.; Zhang, H. Z.; Banfield, J. F.; Elder, S. H.; Zaban, A.; Borse, P. H.; Kulkarni, S. K.; Doran, G. S.; Whitfield, H. J. Energetics of Nanocrystalline $\mathrm{TiO}_{2}$. Proc. Natl. Acad. Sci. USA. 2002, 99, 6476-6481.

12 Lazzeri, M.; Selloni, A., Stress-driven Reconstruction of an Oxide Surface: The Anatase $\mathrm{TiO}_{2}(001)-(1 \times 4)$ Surface. Phys. Rev. Lett. 2001, 87, 266105 1-4.

13 Yang, H. G.; Sun, C. H.; Qiao, S. Z.; Zou, J.; Liu, G.; Smith, S. C.; Cheng, H. M.; Lu, G. Q., Anatase $\mathrm{TiO}_{2}$ Single Crystals with a Large Percentage of Reactive Facets. Nature 2008, 453, 638-641.

14 Thomas, A. G.; Flavell, W. R.; Mallick, A. K.; Kumarasinghe, A. R.; Tsoutsou, D.; Khan, N.; Chatwin, C.; Rayner, S.; Smith, G. C.; Stockbauer, R. L.; et al. Comparison of the Electronic Structure of Anatase and Rutile $\mathrm{TiO}_{2}$ Single-crystal Surfaces Using Resonant Photoemission and X-Ray Absorption Spectroscopy. Phys. Rev. B 2007, 75, 035105 1-12. 
15 Cheng, H. Z.; Selloni, A. Surface and Subsurface Oxygen Vacancies in Anatase $\mathrm{TiO}_{2}$ and Differences with Rutile. Phys. Rev. B 2009, 79, 092101 1-4.

16 Mori-Sanchez, P.; Cohen, A. J.; Yang, W. T. Localization and Delocalization Errors in Density Functional Theory and Implications for Band-gap Prediction. Phys. Rev. Lett. 2008, 100, 146401 1-4.

17 G. Pacchioni, G. Modeling Doped and Defective Oxides in Catalysis with Density Functional Theory Methods: Room for Improvements. J. Chem. Phys. 2008, $128,1825051-10$.

18 Di Valentin, C.; Pacchioni, G.; Selloni, A. Electronic Structure of Defect States in Hydroxylated and Reduced Rutile $\mathrm{TiO}_{2}(110)$ surfaces. Phys. Rev. Lett. 2006, 97, 166803 1-4.

19 Di Valentin, C.; Pacchioni, G.; Selloni, A. Reduced and n-Type Doped $\mathrm{TiO}_{2}$ : Nature of $\mathrm{Ti}^{3+}$ Species. J. Phys. Chem. C 2009, 113, 20543-20552.

20 B. J. Morgan, G. W. Watson. A DFT $+U$ Description of Oxygen Vacancies at the $\mathrm{TiO}_{2}$ Rutile (1 110$)$ Surface. Surface Sci. 2007, 601, 5034-5041.

21 Heyd, J.; Scuseria, G. E.; Ernzerhof, M. Hybrid Functional based on a Screened Coulomb Potential. J. Chem. Phys. 2003, 118, 8207-8215

22 Bylander, D. M.; Kleinman, L. Good Semiconductor Band-Gaps with a Modified Local-Density Approximation. Phys. Rev. B 1990, 41, 7868-7871. 
23 Clark, S. J.; Robertson. Screened Exchange Density Functional Applied to Solids. Phys. Rev. B 2010, 82, 085208 1-11.

24 Guo, Y.; Clark, S. J.; Robertson, J. Calculation of Metallic and Insulating Phases of $\mathrm{V}_{2} \mathrm{O}_{3}$ by Hybrid Density Functionals. J. Chem. Phys. 2014,140, 054702 1-7.

25 Guo, Y.; Robertson, J.; Clark, S. J. The Effects of Screening Length in the Non-local Screened-exchange Functional. J. Phys. Condens. Matter, 2015, 27, $0255011-9$.

26 Gillen, R.; Robertson, J. Hybrid Functional Calculations of the Al Impurity in a Quartz: Hole Localization and Electron Paramagnetic Resonance Parameters. Phys. Rev. B 2012, 85, 014117 1-5.

27 Gillen, R.; Clark, S. J.; Robertson, J. Nature of the Electronic Band Gap in Lanthanide Oxides. Phys. Rev. B 2013, 87, 125116 1-6.

28 Lee, H. Y.; Clark, S. J.; Robertson, J. Calculation of Point Defects in Rutile $\mathrm{TiO}_{2}$ by the Screened-Exchange Hybrid Functional. Phys. Rev. B 2012, 86, 075209 1-8.

29 Janotti, A.; Varley, J. B.; Rinke, P.; Umezawa, N.; Kresse, G.; Van de Walle, C. G. Hybrid Functional Studies of the Oxygen Vacancy in $\mathrm{TiO}_{2}$. Phys. Rev. B 2010, $81,0852121-7$.

30 Malashevich, A.; Jain, M.; Louie, S. G. First-principles DFT Plus GW Study of Oxygen Vacancies in Rutile $\mathrm{TiO}_{2}$. Phys. Rev. B 2014, 89, 075205 1-7. 
31 Zhanpeisov, N. U.; Fukumura, H. Oxygen Vacancy Formation on Rutile $\mathrm{TiO}_{2}$ (110) and its Interaction with Molecular Oxygen: A Theoretical Density Functional Theory Study. J. Phys. Chem. C 2007, 111, 16941-16945.

32 Ortega, Y.; Hevia, D. F.; Oviedo, J.; San-Miguel, M. A. A DFT Study of the Stoichiometric and Reduced Anatase (001) Surfaces. Appl. Surf. Sci. 2014, 294, $42-48$.

33 Zhu, L. G.; Hu, Q. M.; Yang, R. The Effect of Electron Localization on the Electronic Structure and Migration Barrier of Oxygen Vacancies in Rutile. $J$. Phys-Condens Mat. 2014, 26, 055602 1-7.

34 Perdew, J. P.; Burke, K.; Ernzerhof, M. Generalized Gradient Approximation Made Simple. Phys. Rev. Lett. 1996, 77, 3865-3868.

35 Clark, S. J.; Segall, M. D.; Pickard, C. J.; Hasnip, P. J.; Probert, M. J.; Refson, K.; Payne, M. C. First Principles Methods Using CASTEP. Z. Kristallogr 2005, 220, 567-570.

36 Deák, P.; Aradi, B.; Frauenheim, T. Polaronic Effects in $\mathrm{TiO}_{2}$ Calculated by the HSE06 Hybrid Functional: Dopant Passivation by Carrier Self-trapping. Phys. Rev. B 2011, 83, 155207 1-7.

37 Brant, A. T.; Giles, N. C.; Halliburton, L. E. Insertion of Lithium ions into $\mathrm{TiO}_{2}$ (rutile) Crystals: An Electron Paramagnetic Resonance Study of the Li-associated $\mathrm{Ti}^{3+}$ Small Polaron. J. Appl. Phys. 2013, 113, 053712 1-6. 
38 Lany, S.; Zunger, A., Assessment of Correction Methods for the Band-gap Problem and for Finite-size Effects in Supercell Defect Calculations: Case Studies for ZnO and GaAs. Phys. Rev. B 2008, 78, 235104 1-25.

39 Goniakowski, J.; Noguera, C. Electronic-Structure of Clean Insulating Oxide Surfaces .1. A Numerical Approach. Surf. Sci. 1994, 319, 68-80.

40 Majumder, S.; Paramanik, D.; Solanki, V.; Bag, B. P.; Varma, S. Bandgap Tailoring of Rutile $\mathrm{TiO}_{2}(110)$ via Surface Patterning with Electron Cyclotron Resonance Sputtering. Appl. Phys. Lett. 2011, 98, 053105 1-3.

41 Baraff, G. A.; Kane, E. O.; Schluter, M. Theory of the Silicon Vacancy - an Anderson Negative-U System. Phys. Rev. B 1980, 21, 5662-5686.

42 Setvin, M.; Franchini, C.; Hao, X.; Schmid, M.; Janotti, A.; Kaltak, M.; Van de Walle, C. G.; Kresse, G.; Diebold, U. Direct View at Excess Electrons in $\mathrm{TiO}_{2}$ Rutile and Anatase. Phys. Rev. B 2014, 113, 086402 1-5.

43 Deák, P.; Kullgren, J.; Frauenheim. T. Polarons and oxygen vacancies at the surface of anatase $\mathrm{TiO}_{2}$. Phys. Stat. Sol. RRL, 2014, 8, 583-586.

44 Robertson, J.; Clark, S. J. Limits to Doping in Oxides. Phys. Rev. B 2011, 83, $0752051-7$. 


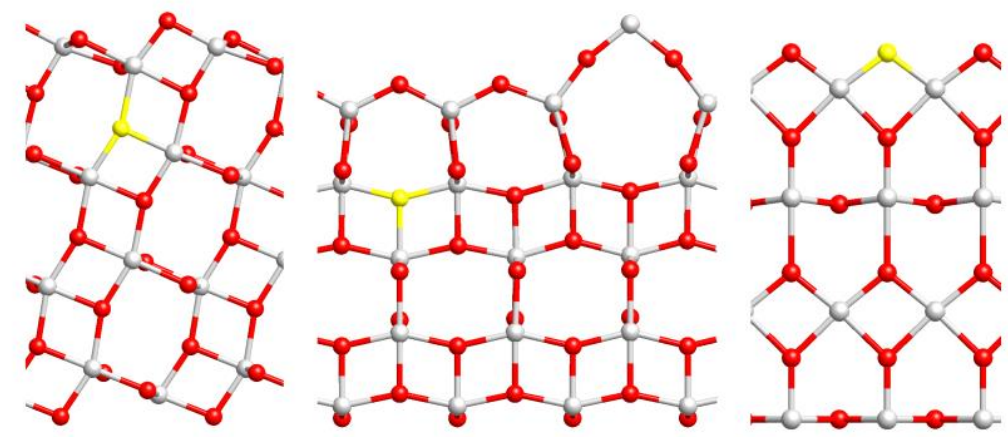

For Table of Contents Only 